\title{
Recent Advances in Nanotechnology: Potential Prospects in Neuromedicine and Neurosurgery
}

\author{
Bassem Yousef Sheikh* \\ Neurosurgery, College of Medicine, Taibah University, Almadinah Almunawarah, Saudi Arabia
}

Received: March 31, 2014; Accepted: June 18, 2014; Published: June 24, 2014

*Corresponding author: Prof. Bassem Y. Sheikh, MD, FRCS, FICS, Professor of Vascular Endovascular Neurosurgery Director, MABL EAJAZ chair for Scientific Research in Prophetic Medicine,Faculty of Medicine, Taibah University, PO Box 456, Almadinah Almunawarah, Zip 41411, Saudi Arabia, E-mail address: askdrsheikh@gmail.com

\begin{abstract}
The advent and popularity of minimal access surgeries and micro-medicine techniques incur the current surge in the utilization of nanotechnology and nanodevices in medical fields. It is obvious that the observed marked increase in nanotechnology research and developments provides a future avenue that portrayed the destiny of neuromedicine and neurosurgery. In fact, it has been the cause for the realization of several potential benefits such as new procedures and therapeutic options for nervous system neoplasia, brain implantation, neuroprotection, nerve nanorepair, efficient drug delivery, nano diagnostic of neurological disease and disorders, nanoimaging, neurological manipulation using nanorobots and nanowires, realtime recording using nanowires arrays and nanocircuits etc. Certainly, nanotechnology is providing a future platform for further research and development in neuroscience and neurosurgery.

However, despite the significant advances provided by nanotechnology in terms of neurosurgical procedures, modern neuroimaging and contrast media; still some significant limitations exist. Thus, the applications of nanotechnology in the field of neurosurgery and neuroscience need to be precisely understandable for more transparent and honest solutions to foster their medical potentials. This review examines the recent developments and potentials of the utilization of nanotechnology in Neuromedicine and Neurosurgery.
\end{abstract}

Keywords: Nanotechnology; Neurosurgery; Glioblastoma; Nanoparticles; Brain; Brain Implant; Nanowires; Nanorobots; Photodynamic Therapy; Quantum Dots

\section{Introduction}

The pathological correlation of brain lesions that led to the diagnosis of central nervous system (CNS) neoplasia, vascular lesions, and spinal disorders were documented to be due to the progressive affirmation of Neuroscience and Neurosurgery. The introduction of ventriculogram, and angiographyin the second and third decade were among the reported techniques that provided the possibility for indirect diagnosis of brain tumors and vascular pathologies. ${ }^{[1]}$ Despite several efforts which were made to curtail the risk associated with the neurosurgical procedures, still the mortality and morbidity of neurosurgical disease were said to be profoundly high. The increasing need of specialized and intense neurosurgical operative procedures of higher sophistication, lack of skilled neuro-anaesthesia, difficulty in drug delivery across blood brain barrier (BBB), and lack of microsurgical instruments among other things were considered to be among the reasons of the associated mortality and morbidity. This led to the urgent need for alternative therapeutic options in neuromedicine and neurosurgery.

Among the exploited options include the use of alternative therapy, ${ }^{[2,3]}$ as well as invasive devices and novel surgical techniques that could be employed in neurosurgery. For example, endoscopy was introduced into neurosurgery practice for more than 20 years, but difficulty was in the safe application. This continued to be an obstacle until the reporting of an excellent instrumentation in the holding of endoscope was briefly described. ${ }^{[4]}$ Similarly, performing of cerebral angiogram during neurosurgical procedure was held as highly risky. This was improved dramatically by the noble description of minimal invasive technique for cerebral angiogram during a neurosurgical procedure. ${ }^{[5]}$

The continued advances in research on bio-electronic engineering and biophysics resulted in the development of Computed Tomography (CT) scan, which helped a lot to delineate brain lesions in a more precise form; which off course, no doubt, improve the comfort level of the neurosurgeon. The introduction of magnetic resonance imaging (MRI) and the invention of the high magnification surgical microscope were said to be among the sources in the observed improvement in the surgical resection of brain CNS neoplasia. ${ }^{[1]}$ While investigation concerning the safety of surgical materials were also not left behind ${ }^{[6]}$ and in event of brain death, the transcranial doppler was said to be used as a confirmatory test. ${ }^{[7]}$

The advent and popularity of minimal access surgeries and micro-medicine techniques incur the current surge in the utilization of nanotechnology and nanodevices in medical fields. The application of nanotechnology to the medical field resulted in a new frontier in medicine described as "nanomedicine", which is the exploitation of structured nanoparticles such as dendrimers, ${ }^{[8]}$ carbon fullerenes, ${ }^{[9,10]}$ and nanoshells, ${ }^{[11]}$ to target specific tissues 
and organs in applications such as medical imaging, ${ }^{[12]}$ nerve regeneration, ${ }^{[13]}$ CNS neoplasia targeting etc. ${ }^{[14,15]}$ Nowadays, with the aid of nanodevices that serves as contrasting agents, maximal safe resection of the brain tumours such as gliomas and pituitary adenomas are achieved via rendering using intraoperative MRI imaging technolog. ${ }^{[1]}$ These kinds of nanoparticles can be effectively transported across BBB. They were reported to demonstrat early preclinical success in management of CNS neoplasia, acute ischemic stroke, Alzheimer's disease, and HIV encephalopathy. ${ }^{[16]}$

The current surge in biotechnological research has lent a momentum to the observed development in Neuro-modulation using stem cells for neurological functional restoration in patients with spinal cord injury and certain degenerative neurological diseases. Genetic microarrays could be potential in diagnosing the type of CNS neoplasia and then suggest relevant molecular targeted therapies.

The nanoscale nature of nanoparticles and their noninvasive nature during several procedures incurred their influence in various areas of research and development. ${ }^{[17]}$ The nanoparticles properties are exploited for their reported light-refractive, magnetic, and biological properties; whereas their conjugate with other bioactive ligands was reported to incur their specificity and solubility. ${ }^{[18]}$ Among the potential applications of nanotechnology in neurosciences include nerve nano-repair, ${ }^{[19]}$ nano-imaging with nano-particles and quantum dots, ${ }^{[20,21]}$ manipulation of CNS with surgical nanobots, ${ }^{[22]}$ and nano-neuromodulation with nano-fibres and nano-wires. ${ }^{[23,24]}$

In fact, the nature of this material was reported to allow for the development of nanodevices that are extensively utilized at subcellular levels with higher accuracy in cell-specific targeting in biomedical applications, resulting in efficient therapeutic results with minimal or benign effects. ${ }^{[17,25]}$ This recent development in the field of nanoscience and nanoparticle formulations leads to the development of several novel nanodevices that found applications across all fields.

Considering its potential applications, it suffice that nanotechnology will have a broad and fundamental impact on many sectors of the global economy. Currently, the reported global market for nanotechnology was valued at nearly $\$ 20.1$ billion in 2011, and it has been projected to reach $\$ 48.9$ billion in 2017.[26] Moreover, sales earnings from nanomaterials and nanodevices alone, were anticipated to reach 37.3 and 11.4 billion US dollars in 2017, respectively. ${ }^{[26]}$

Thus, the applications of nanotechnology in the field of neurosurgery and neuroscience need to be precisely understandable for more transparent and honest solutions to foster their medical potentials. This article aims to explore the recent advances on nanotechnology and nanodevices that are exploited in various avenues specifically in applications pertaining neuromedicine and neurosurgery based on instrumentation and applied procedures.

\section{Nanotechnology in neuro-drug delivery}

The versatile designs in nanoparticles fabrication made them to be utilized in several applications. Among other employed techniques, nanoparticles can be engineered to move desired molecules or compounds into target locations by using electrostatic forces, bioactive ligand conjugation, or convection enhanced delivery.

Recent advances in nanotechnology have paved the way for efficient delivery of therapeutic drugs to brain and across the blood brain barrier (BBB). Drugs that are known to be poorly distributed in the brain parenchyma were said to have higher drug bioavailability when loaded onto a nanocarrier system that could interacts well with the endothelial micro-vessel cells. These nanocarriers were known to further be functionalized (conjugated) with targeting ligands (e.g. folic acid) to selectively bind to putative receptors or transporters expressed at the BBB for enhanced CNS selectivity and permeability. ${ }^{[27]}$ Solid lipid nanoparticles (SLNP) are generally a matrix of physiological lipids (fatty acids, glycerides, glycerine, waxes and their mixture), that remain solid at room or body temperature. SLNP were used as cancer drugs carriers to efficiently deliver the drugs to the brain. ${ }^{[28,29]}$ Another important recent application is in Alzheimer's disease, that is a progressive age-related neurodegenerative disorder that is associated with distinct neuropathological features. The disease was said to be inflicted in about three percent of world's population between the age of 65 to 74 years, and nearly half of the population aged 85 years or older. ${ }^{[30]}$ Quercetin, a phytochemical flavonoid, was said to be a novel neuroprotectant by inhibiting the increased levels of reactive oxygen species produced by mitochondrial activity, which are known to accelerate the neurodegenerative processes of Alzheimer's disease. ${ }^{[31]}$ Dhawan et. al. ${ }^{[32]}$ reported an enhanced delivery of quercetin into the brain by encapsulation in optimized formulations of SLNP that were prepared by microencapsulation technique using compritol as the lipid and Tween 80 as the surfactant. The researchers were able to encapsulate the quercetin into the SLNP by first melt-mixing the lipid and the quercetin at $70-75^{\circ} \mathrm{C}$. this mixture was then added to an isothermal aqueous mixture of water and surfactant under continuous stirring and incubated in an ice-bath. ${ }^{[32]}$ Depending on experimental conditions, the researchers reported as high as $90 \%$ in vitro drug release; and in vivo behavioral and biochemical experiments revealed markedly better memory-retention in rats treated with the encapsulated quercetin compared to those treated with free quercetin. ${ }^{[32]}$ Glioblastoma multiforme (GBM) is regarded as the most aggressive brain carcinoma and the most malignant form of astrocytoma. In fact, the disease is among the most commonly encountered CNS glioma, and is categorized as grade 4 glioma. ${ }^{[33]}$ Taking the advantage of glioma's expression of certain types of growth factor receptors, target specific drug carriers were designed to deliver potent drugs directly to glioma cells achieving a focused therapeutic strategy. Recently, Kuo and Liang ${ }^{[34]}$ human brain malignant glioblastoma cells were inhibited using carmustine encapsulated in cationic SLNP tagged with surface anti-epithelial growth factor receptor(Figure 1a). During the nanoparticles formulation, the authors revealed that the minimal average diameter of the nanoparticles and maximal entrapment efficiency of fluorescent-labeled carmustine was observed when the cationic surfactants of $1 \mathrm{mM}$ concentration 
was used . Furthermore, they described the nanoparticle to exhibit an effective delivery of the drug to U87MG cells and antiproliferative efficacy against the growth of malignant brain tumors in a concentration dependent manner. ${ }^{[34]}$

Similarly, brain capillaries present at BBB were reported to be the main obstacles for the drug delivery to the brain, due to restriction by p-glycopro-tein (p-gp) efflux. ${ }^{[35]}$ Co-delivery of p-gp inhibited drug and a drug that doesn't suffer the effect was hailed as an alternative method to efficiently deliver the drug to brain across BBB. The p-gp efflux is known to restrict the efficient delivery of docetaxel (a widely used anticancer drug) to the brain across BBB. ${ }^{[35]}$ Recently, a dual drugs of docetaxel and ketoconazole, a synthetic imidazole, which is known to inhibit $\mathrm{p}$ gp efflux were successfully encapsulated in SLNP that were conjugated with folate (to target cancer expressed follate receptor protein) used to efficiently deliver the drug's cocktail across BBB (Figure 1b). In the study, the authors found the use of this dual drug in SLNP to increase brain uptake of docetaxel with 44 times higher brain permeation coefficient (Kin) than that of Taxotere. ${ }^{[35]}$ Several litetures reported the used of different forms of SLNP for efficient delivery of drugs to brain and other part of CNS, ${ }^{[36-38]}$ as summarized in Table 1 .

The high surface area of nanoparticles provides an ample place for placing cellular and active protein targeting probes, which inturn overcome the low selectivity of the anticancer drugs towards the cancer cells. Beside the use of SLNP, researchers have employed the use of conjugated lipid nanocapsules (a capsule made up of a mixture of triglycerides and phospholipids) to also deliver drug to the CNS. ${ }^{[4]}$ Solvent free phase invation process was used to fabricate docetaxel loaded lipid nanocapsules that were used to deliver drug to solid tumors in mice. ${ }^{[46]}$

Recently, it has been reported that 14.6 months are the median survival time for glioma patients treated with radiotherapy plus temozolomide, whereas 12.1 months were reported to be that of glioma patiants treated with radiotherapy alone. ${ }^{[47]}$ Thus, necessitating the development of new and effective therapies. ${ }^{[48]}$ Among the highly considered drug candidate is the tamoxifen, a member of selective estrogen receptor modulator (SERM) family, which showed a promising results in treating patients with recurrent malignant gliomas based on high dosage trials. Allard et. al. ${ }^{[48]}$ delivered ferrocenyl diphenol tamoxifen (an organometallic tamoxifen derivatives) encapsulated in lipid nanocapsule (LNC) to $9 \mathrm{~L}$ glioma model. The research revealed the procedure to significantly lower both tumour mass and volume evolution of the implanted 9L-cell in rats by high intake of the drug (Figure 2). Looking into other therapeutic dimensions, internal radiotheraphy is condisered as an alternative, complement to, managment of malignant gliomas. Allard et. al. ${ }^{[49]}$ reported the use of LNC to entrapped lipophilic complexes of 188Re, which were used as internal radiotherapeutic agent agianst malignant 9L glioma cells by means of convection-enhanced delivery. Similar studies using LNC conjugated with OX26 monoclonal antibodies were also reported. ${ }^{[50]}$

Liposomes are small vesicles madeup of either uni or
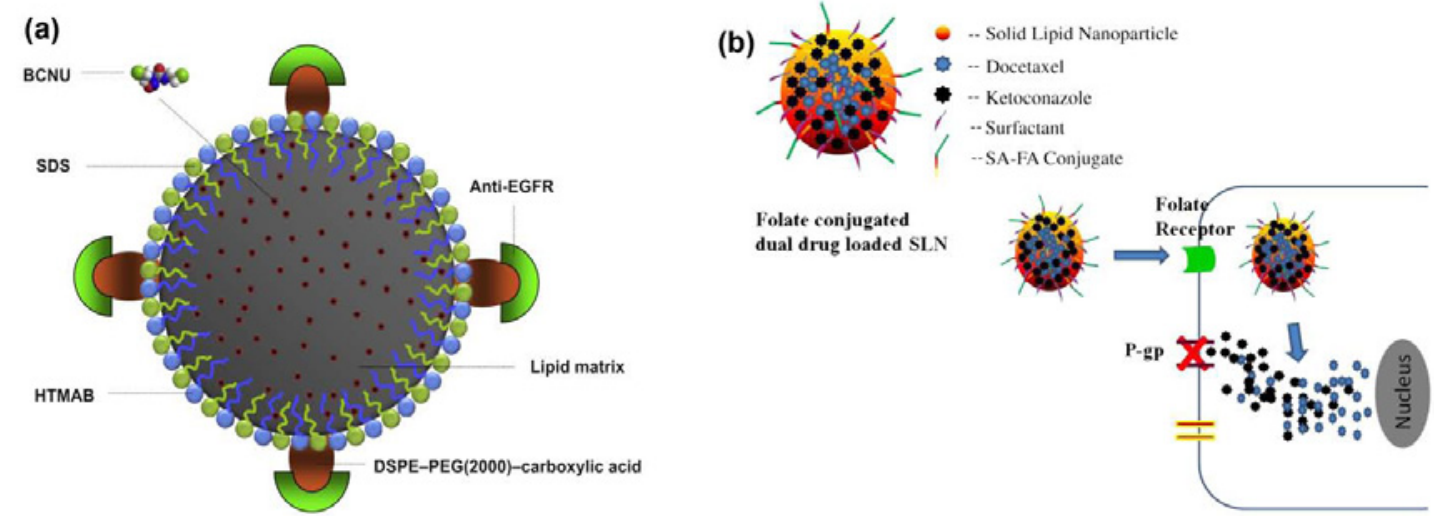

Figure 1: (a) Illustrative carmustine-loaded cationic solid lipid nanoparticles labelled with surface anti-epithelial growth factor receptor for targeting glioma cells. (b) Folate conjugated dual drug carrier for efficient delivery across BBB. Adopted from ${ }^{[34]}$ and ${ }^{[35]}$, with permission from Elsevier.

Table 1: Reported nanoparticles used as drug carriers to CNS.

\begin{tabular}{|c|c|c|c|}
\hline Nanocarrier type & Drug delivered & Target disease & References \\
\hline SLNP & Curcumine & Alzheimer's disease & {$[39]$} \\
\hline SLNP & Risperidone & Psycotic disoders & {$[40]$} \\
\hline Cationic SLNP & Etoposide & CNS malignant tumors & {$[41]$} \\
\hline LNC & Ferrociphenol & Intracranial 9L glioma & {$[42]$} \\
\hline Liposomes & Daunorubicin & C6 glioma & {$[43]$} \\
\hline Dendrimers & Doxorubicin & C6 glioma cells & {$[44]$} \\
\hline
\end{tabular}


multilamellae phospholipid-lipid bi-layer with an internal compartment that is used to encapsulate therapeutic drugs. Their ability to deliver both hydrophilic and hydrophobic drugs as well as long tracking record, with low toxicity and high bioavailability; made liposomes to be one of the extensively exploited nanodrug carriers in nanomedicine. The conjugated targeting ligands have been used in several drug carriers to target specific receptor in order to enhance the drug delivery to the cells in CNS. Many ligands were said to be successful, but it is difficult to compare the results from different studies to determine which targeting ligand is more efficient. Five different targeting ligands (transferrin, RI7217, COG133, angiopep-2, and CRM197) were studied and compared based on their ability to target liposomes to the brain in both in vitro and in vivo. ${ }^{[51]}$ The study showed that of all the targeting ligand tested, only the RI7217 was able to significantly enhance brain uptake in vivo throughout the experimental time course. Furthermore, conjugating the liposomes with targeting ligand resulted in a respective 10 times and 4.3 times higher uptake in the brain capillaries and parenchyma compared to untargeted liposomes.

A dendrimers consisting of $\mathrm{pH}$-sensitive dual-targeting drug carrier was synthesized based on poly (amidoamine) carrying targeting ligand transferrin and encapsulating tamoxifen were efficiently transported across BBB, resulting in higher accumulation of tamoxifen in the glioma cells. ${ }^{[52]}$ Similar study reported the use of biotin conjugated dendrimers based on poly (amidoamine) were also used to enhanced drug delivery to the brain across the BBB. ${ }^{[33]}$

\section{Functional nanaoparticles for diagnostic Imaging}

To better define nature and internal geometry of neurological lesions from their surrounding tissue, computer tomography (CT) imaging techniques were usually observed with the aid of enhancing contrast materials such as iodinated compounds. ${ }^{[54]}$ The contrast materials help to manipulate the density of the intravascular space, thereby providing a contrasted imaging of the lesions. The radiographic hyperdensity of iodinated contrast materials against normal brain tissue and cerebrospinal fluid made it possible to outline the vascular anatomy when introduced intravenously. ${ }^{[18]}$ Among the hypervascular lesions that were reported to show greater contrasting imaging upon iodinated contrast on CT include meningioma and arteriovenous malformations. ${ }^{[18]}$ Unfortunately, the half-life of iodinated contrast was reported to be in the order of two hours, and the peak iodine levels fall within 5-10 minutes. ${ }^{[55]}$ Thus, requiring repeated infusions when multiple imaging is needed. Although, the metabolic products of iodinated contrast media can totally be excreted renally. In this situation, patients with renal failure will retain elevated iodine levels for a long period of time resulting in "contrast-induced nephropathy", which was reported to be a lifethreatening condition with about $14 \%$ morbidity and mortality incidence in high-risk patients such as those with diabetes or renal malfunctions. ${ }^{[18]}$ Recently, gold nanoparticles were also reported to be employed as contrast enhancing materials in the diagnosis of intracerebral malignant glioma. ${ }^{[56]}$

One of the earliest applications of nanoparticles in nanomedicine is the use of Magnetic Resonance Imaging (MRI) in neuro-pathological diagnosis. Like CT, contrast materials were also reported to be employed in this technique. For over two decades, ferromagnetic nanoparticles such as paramagnetic iron oxide and gadolinium based particles were known to be used as MRI contrast materials when taken up by the host. These particles were known to have the potential to help the neurosurgeon to distinguish between normal and tumor cells. In particular gadolinium-based contrast material is said to utilize the magnetic dipole of the metal to produce a weak paramagnetic effect resulting in a hyperintense signal on MR machine ${ }^{[18]}$ However, just like iodinated contrast materials, gadolinium-based contrast materials were reported to have similar limitations of distribution half-life of approximately 12 minutes and an elimination halflife of 1.6 hours and are not biocompatible. ${ }^{[55]}$ In addition, a gadolinium-based contrast material does not cross intact BBB, but rather cross pathologically defective BBB to help in acquiring the lesions geometry for various neuro-pathologies. It is also not readily excreted renally, thence resulting in nephrogenic systemic fibrosis in patients with severe renal insufficiency. ${ }^{[57,58]}$

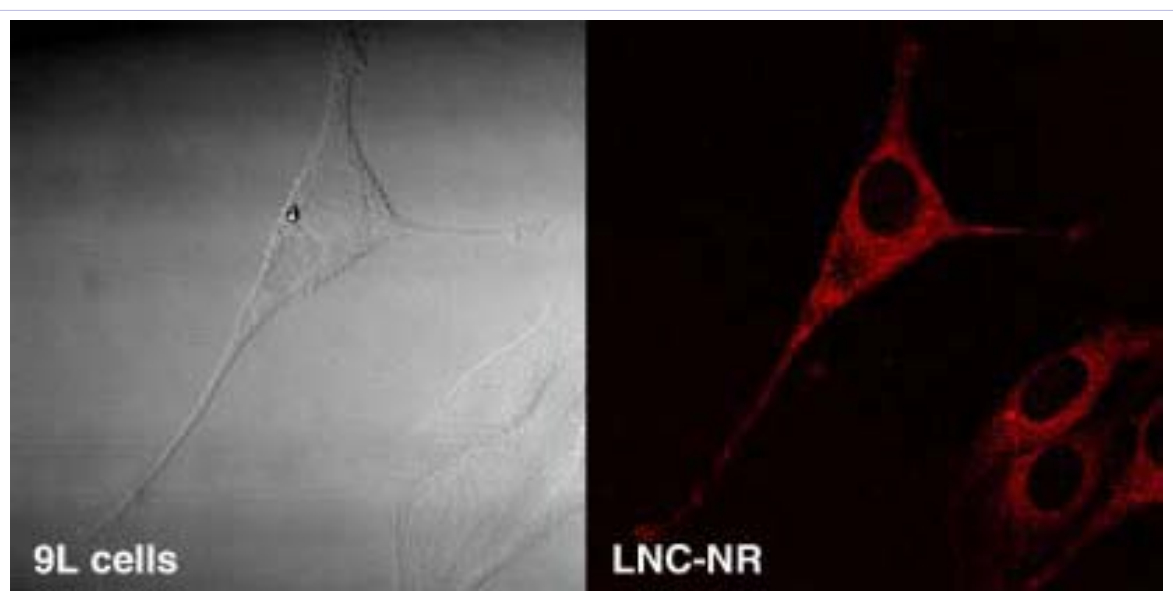

Figure 2: Confocal micrographs showing the $9 \mathrm{~L}$ glioma cells and the uptake of red flourecent-labelled tamexifone loaded nanocapsules in the cells. Adopted from ${ }^{[48]}$, with permission from Elsevier. 
Unlike gadolinium-based contrast agents, ferromagnetic contrast nanoparticles are biocompatible and can be processed by host cell's via iron related metabolic pathways. The enhancement of rat glioma MRI after intravenous administration of iron oxide particles were reported in several studies. ${ }^{[59-61]}$ Furthermore, these particles when coated with dextran, affords uncomplicated bonding of ligands or functional groups and facilitated uptake through different cellular transport systems. Unfortunately, due to the high affinity and uptake of ferromagnetic contrast nanoparticles by the reticuloendothelial system resulting in their entire sequestration by splenic, hepatic and lymphatic tissue, the neuroimaging applications of standard ferromagnetic nanoparticles is still of high limitation. ${ }^{[18]}$ However, surface modification of these molecules by conjugation with polyethylene glycol (PEG) is said to inhibits uptake by the reticuloendothelial system, thus, prolongs circulation, promoting delivery to neural tissue by penetrating BBB. ${ }^{[62,63]}$

Previously, nanoparticles were demonstrated to have an improved crossing BBB into the white matter of rats with experimental allergic encephalomyelitis due to PEGylated polyalkylcyanoacrylate conjugation compared with nonPEGylated nanoparticles. ${ }^{[62]}$ Another proven effective approach in delivering nanoparticles across an intact BBB is by chelating bioactive molecules such as diethylenetriamine pentaacetic acid to the contrast material to mediate endocytosis by brain capillary endothelial tissue. ${ }^{[64]}$

A dextran-coated iron oxide nanoparticle (Ferumoxtran-10) in ultrasmall superparamagnetic nanoparticles of iron oxide (USPIO) was used to provide enhanced MRI images (Figure 3) in patients with intracranial malignant tumors. ${ }^{[64]}$ Similarly, ferumoxtran-10 was loaded onto USPIO were used as magnetic resonance contrast agents to acquire a contrasting images of brain tumor.

A detailed description of the model for the mechanism of actions of both gadolinium and ferumoxtran- 10 contrast materials on crossing the BBB to reach the brain's extracellular matrix and lesion cells during MRI was proposed. ${ }^{[64,65]}$ The researchers described that the ferumoxtran-10 nanoparticles that crosses the defective $\mathrm{BBB}$ are phagocytize by the macrophages and reactive astrocytes in and around the lesion, entrapping them for about 5-7 days permitting visualization by MR during this period. ${ }^{[64,65]}$ As the entrapment time prolongs, the particles are then degraded inside the phagocytic cell's lysosomes to free irons (Figure 4), which are release to the iron-transporting proteins of the general circulation and stored with the rest of the body's iron reserves. [65]

A multifunctional nanoparticles (30-60 nm) containing conjugated photosensitizer were employed to deliver reactive oxygen species (ROS) to cancer cells while simultaneously enhancing MRI contrast providing real-time tumor kill measurement. ${ }^{[66]}$ Since Kopelman description of multifunctional nanoparticles, several literatures reported a similar exploitation to achieve a simultaneous drug delivery and tumor diagnostic imaging at a time. ${ }^{[67-71]}$ Due to the limitations of the current imaging techniques on inadequate sensitivity, and spatial resolution, coupled with the difficulty in displaying or obtaining a contrasted tumor-margins are among the major obstacles in the path of obtaining precise tumor geometry and boundary toward better outcomes for patients with brain tumors. This limitation was recently overcame by using a unique triplemodality MRI photoacoustic-Raman (MPR) imaging technique to delineate the tumor margin in rat brain at both preoperative and intraoperative stages. A Raman molecular tag (trans-1,2-bis(4pyridyl)-ethylene) was used by the researchers to cover a gold core nanoparticle of about $60 \mathrm{~nm}$, thereafter, the raman outer layer was protected by a $30 \mathrm{~nm}$ silica coating. The nanoparticle was then conjugated with modified gadolinium containing 1,4,7,10-tetraazacyclododecane-1,4,7,10-tetraacetic acid (GdDOTA) resulting in gold-silica-based SERS nanoparticle coated with Gd3+ ions, otherwise described as the MPR nanoparticles (Figure 5). The researchers then developed an orthotopic brain tumor model in mice by implanting using a transfected human glioblastoma cells conjugated with an enhanced green fluorescent protein (eGFP+U87MG) into the striata of nude mice using a stereotactic implantation device. ${ }^{[72]}$ The tumoral mice were intravenously injected with matragel diluted MPR nanoparticles via tail veins, and the mice were scanned using the MRI, photoacoustic and Raman systems (Figure 5).
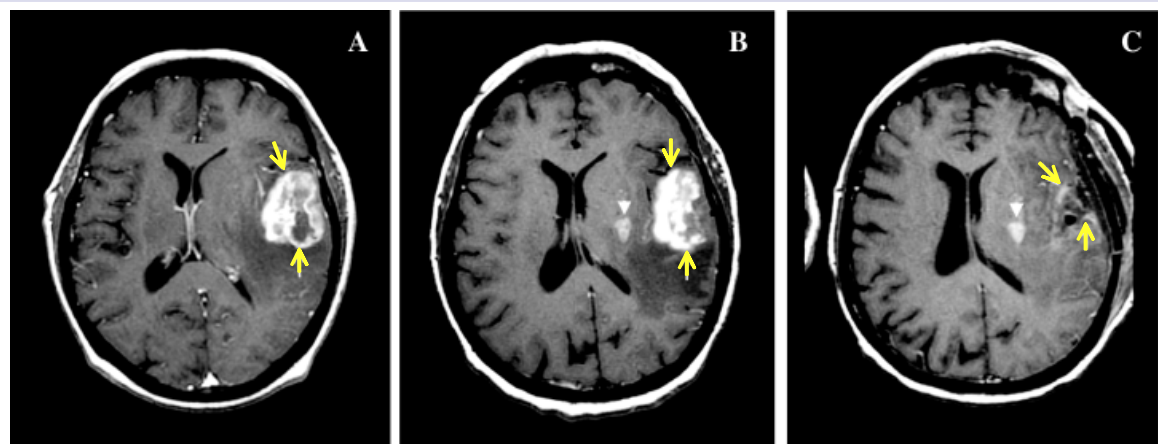

Figure 3: MRI images of A. Gadolinium-enhanced spin-echo (SE) T1-weighted image shows a large left fronto-parietal enhancing tumor (arrows). B. At $24 \mathrm{~h}$ after ferumoxtran infusion, preoperative SE T1-weighted image demonstrates intense ferumoxtran enhancement in the left fronto-parietal tumour (arrows). C. At 5 days after ferumoxtran infusion and 4 days after surgery, postoperative SE T1-weighted image reveals still clear visualization of the residual ferumoxtran-enhancing lesion in the putamen (arrowhead); Adopted from ${ }^{[64]}$ [64], with permission from Wiley online library. 


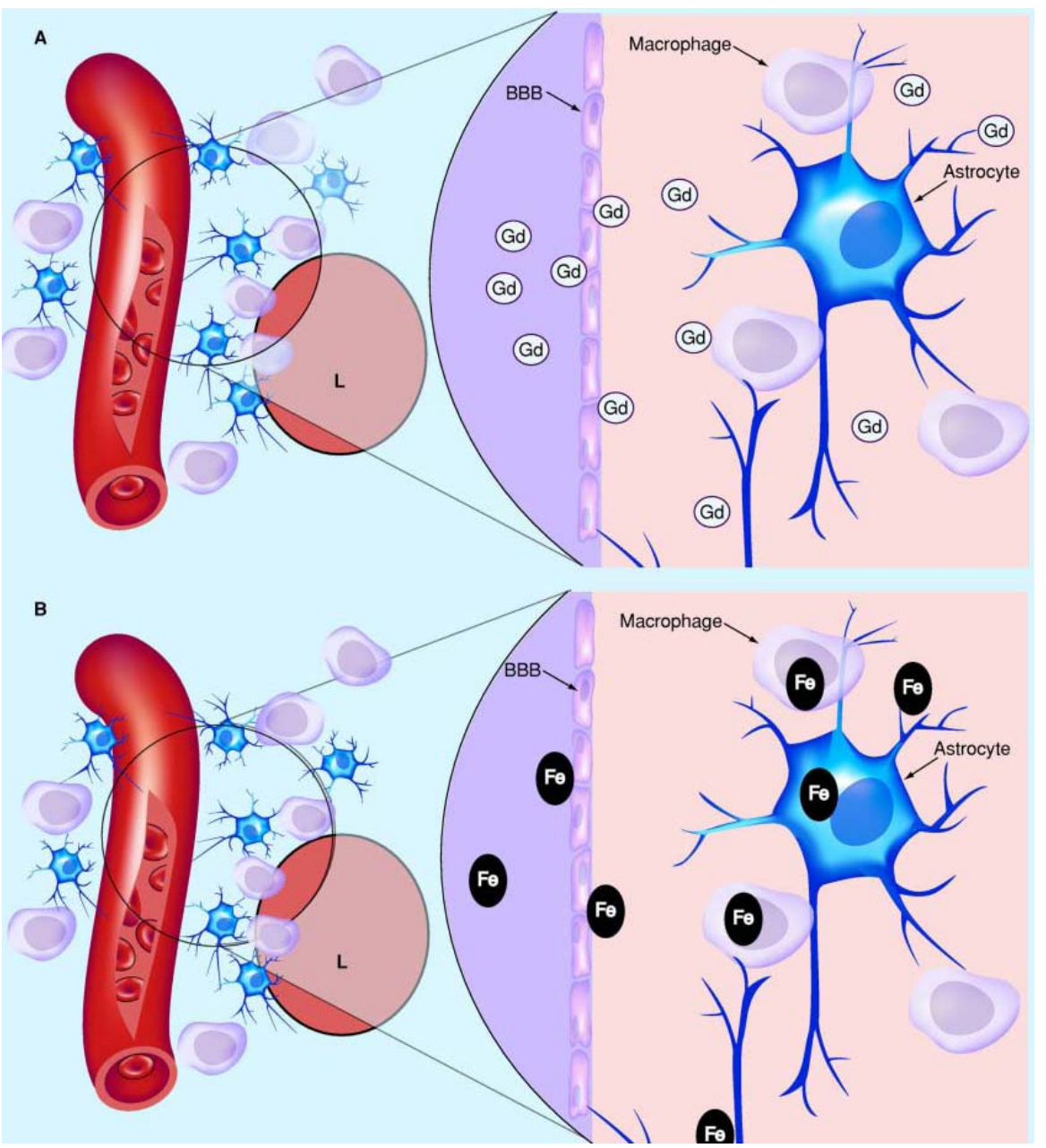

Figure 4: MR contrast enhancing material crossing the defective BBB, (A) Gadolinium (Gd) chelates crossing from the intravascular compartment through a defective BBB (arrow) to the interstitial space. Gd remains in the extracellular space. Macrophages and reactive astrocytes are shown around the lesion (L) but Gd does not enter these cells. During its short half-life (1.6 h) Gd rapidly crosses the BBB to produce a hyperintense signal on T1-weighted sequence on MR. (B) Ferumoxtran-10 (Fe in black) and other ultrasmall iron oxides are much larger particles compared with Gd and only slowly cross the defective BBB (arrow). Due to its prolonged half-life (24-30 h), particles of ferumoxtran-10 will progressively accumulate in the interstitial space where they will be endocytosed by reactive astrocytes and macrophages, remaining there for up to 7 days. Adopted from ${ }^{[65]}$.

Furthermore, their study revealed that the scanned in vivo signals are highly correlated to the MPR concentrations in both MRI, photoacoustic and Raman analysis as shown in Figure 6. ${ }^{[72]}$ Similarly, a multi-modal nanoparticle probe termed Cy5.5-CLIO were used as MRI contrast agent to delineate the brain tumor margins and the technique was reported afforded an intraoperative discrimination of tumors from brain tissue because of the particle's near-IR fluorescence. ${ }^{[73]}$ More recently, fabricated nanoparticles such as quantum dots, ${ }^{[74,75]}$ liposomes, ${ }^{[76,77]}$ dendrimers, ${ }^{[78,79]}$ nanoshells, ${ }^{[80,81]}$ nanotubes, ${ }^{[82,83]}$ nanoemulsion, ${ }^{[84,85]}$ and viruses ${ }^{[86]}$ have been developed as imaging agents in nuerological tumor diagnosis.

\section{Nanodevices in neurological surgery procedures}

Micro-fabricated devices with nanoscale features have been proposed as new micro-instrumentation for cellular and subcellular surgical procedures. Carbon nanostructures have excellent mechanical, electrical, and conduction properties, and have nanostructure similar to neuritis. ${ }^{[13,83]}$ Hence, they have been utilized to improve neural activities and guide severed ends in a nerve through each other. ${ }^{[9,24]}$ Chemical functionalization can produce various surface charges on MWCNTs which in turn control neural growth. It has been reported that Activation of the phospholipase $\mathrm{C}$ signals pathway in nerve growth factor-treated neurons by carbon nanotubes. ${ }^{[87]}$

The recent surge in nanotechnological advancement resulted in the development of noninvasive minimal access surgery. Thanks to the nanorobotics technology, which leads to the fabrication of nanorobots that were said to be injected into the patient to perform diagnosis or treatment on a cellular nanoscale level. ${ }^{[88]}$ Magnetic nanorobots were used to safely deliver drugs across BBB via locally hyperthermic disruption of the BBB by induction of MNPs inside an AC field. ${ }^{[89]}$ The efficiency of the delivery of such kind of nanorobot is largely said to depends on the precision of the attached or conjugated motion controllers. 


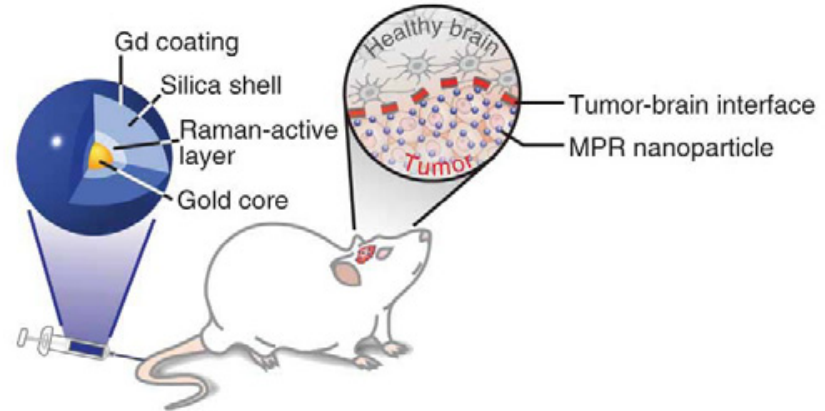

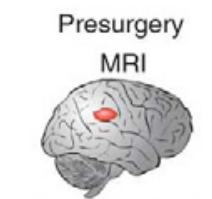

Surgical planning

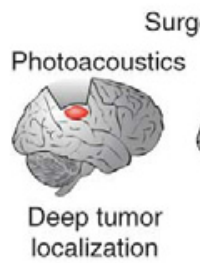

Surgery

Postsurgery

Raman

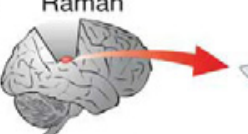

Fine margin

resection

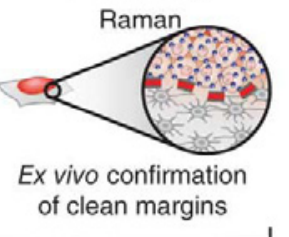

Injection

day (0)

Figure 5: Schematic diagram of triple-modality MPR concept. MPRs are injected intravenously into a mouse bearing an orthotopic brain tumor (top). As the nanoparticles circulate in the bloodstream, they diffuse through the disrupted blood-brain barrier and are then sequestered and retained by the tumor. The MPRs are too large to cross the intact blood-brain barrier and, therefore, cannot accumulate in healthy brain. Adopted from ${ }^{[72]}$, with permission from Nature publishing group.
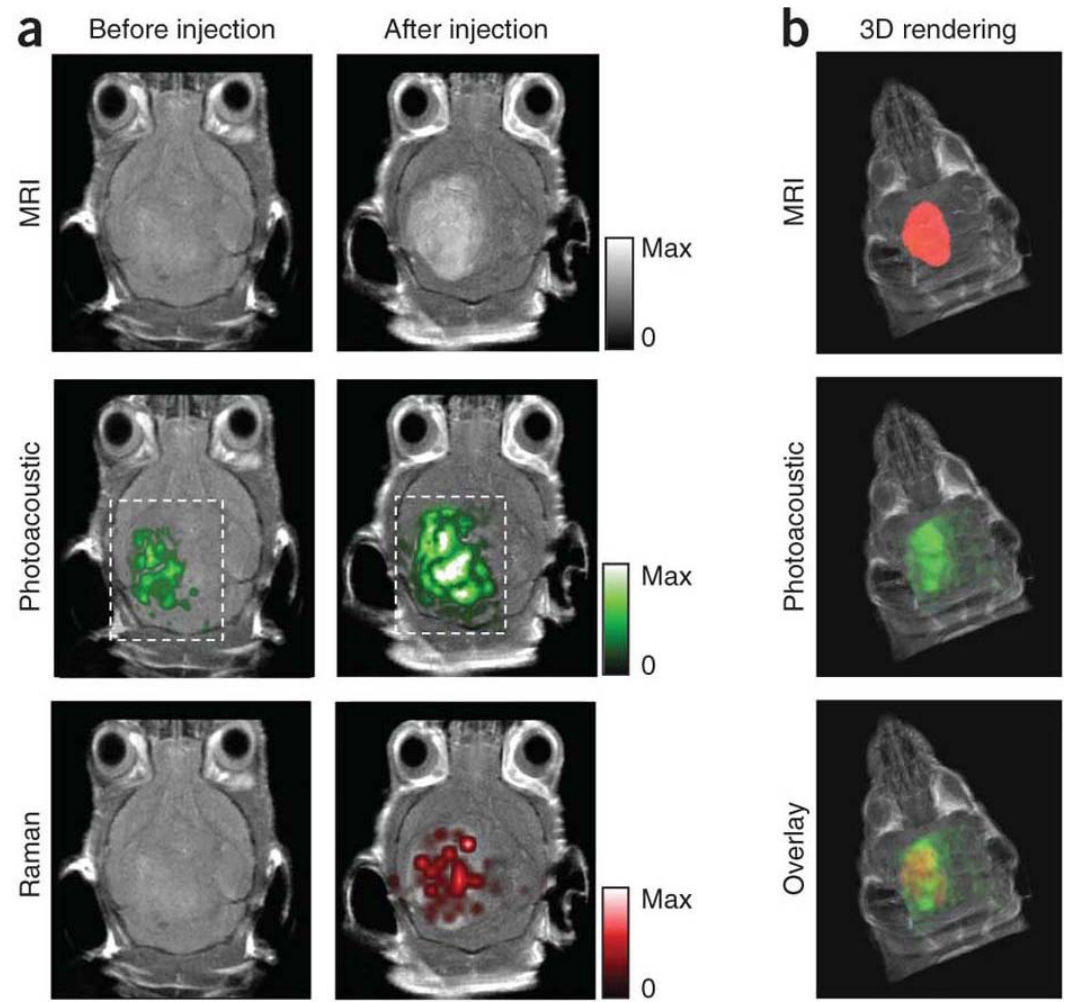

Figure 6: (a) Two-dimensional axial MRI, photoacoustic and Raman images. The post-injection images of all three modalities showed clear tumor visualization (dashed boxes outline the imaged area). (b) A three-dimensional (3D) rendering of magnetic resonance images with the tumor segmented (red; top), an overlay of the three-dimensional photoacoustic images (green) over the MRI (middle) and an overlay of MRI, the segmented tumor and the photoacoustic images (bottom) showing good colocalization of the photoacoustic signal with the tumor. Adopted from ${ }^{[72]}$, with permission from Nature publishing group.

Citation: Sheikh BY (2014) Recent Advances in Nanotechnology: Potential Prospects in Neuromedicine and Neurosurgery. Nanosci Technol 1(2): 1-13. 
${ }^{[90]}$ Employing this hypothesis, a magnetotactic bacterium (MTB) is controlled by orienting the magnetic fields towards a target positioning order to effect enhanced drug delivery. ${ }^{[91]}$ Two photon polymerization were used to fabricate a controllable actuated swimming nanorobots made of a magnetic polymer composite (MPC) containing magnetite nanoparticles of $11 \mathrm{~nm}$ in diameter and photo-curable resin. ${ }^{\left[{ }^{[2]}\right.}$ Similarly, neuro-invasive multi-targeted drug delivery was achieved using a swarm of magnetic nanorobots carriers. ${ }^{[93]}$ Similar reports on drug delivery using nanorobots were described elsewhere. ${ }^{[94,95]}$

Regarding noninvasive and the difficulty experienced by neurosurgeons during tumor surgery, the use of nanosurgery are hailed as an excellent application of nanotechnology to the field of neuromedicine. During this kind of surgical procedure the tumors are manipulated or extinguished by directing beams of forceful fast laser beams that are focused by an objective microscope lens. Ben-Yakar and Bourgeois ${ }^{[96]}$ reported the used of ultrafast laser nanosurgery for in vivo nerve regeneration studies. Femtosecond laser pulses was shown to provide the novel capacity for precise, sub-surface, cellular-scale cuts for neurosurgical applications in rats. ${ }^{\left[{ }^{[9]}\right.}$ In addition, a novel photodisruption technique described as Plasmonic Laser Nanosurgery (PLN) was used to exploits the large enhancement of ultrafast laser pulses in the near-field of gold nanoparticles for the nanoscale manipulation of biological structures ${ }^{[98]}$ Recent procedures concerning clinical laser surgery were reviewed in some literatures. ${ }^{[99,100]}$

For over five decades, modified insulated microelectrodes were known to be employed in neurophysiological studies. [101] Previously in our laboratory, we have reported the use of siliconsubstrate microelectrode arrrays implanted in cerebral cortex for chronic brain cortical recording. ${ }^{[102]}$ Qiao et. al. ${ }^{[103]}$ demonstrated that $700 \mathrm{~nm}$ tipped electrode recorded wellisolated action potentials extracellularly from single visual neurons in vivo. Further experimental studies using sub-100 nm silicon nanowires revealed its integration into live cells without causing detrimental effects. ${ }^{[104]}$ Transistor arrays of silicon nanowires with diameter of $30 \mathrm{~nm}$ that were fabricated on transparent substrates were used to map neural circuits in acute brain slices Qing et al. 2010 The neural circuit is said to provides an avenue that can record across a wide range of length scales, while the transparent device chips only provide imaging of individual cell bodies. ${ }^{[101]}$ Recently, Robinson et. al. ${ }^{[105]}$ described a scalable platform for intracellular interfacing to neuronal circuits using vertical nanowire electrode arrays. The combination of arrays with patch clamp was used to record the activity with high temporal and spatial resolution, as well as mapping of functional connectivity, providing a powerful platform for studying neural circuits in the brain.

Precision is considered to be one of the outmost important factors in surgical procedures including neurosurgery, and the robotics contribution of nanotechnology has introduces another dimension in the field of neurosurgery. Nanorobots miniaturized nanoscale devices fabricated from active nanomaterials such as synthetic zinc oxide, gold, quartz etc. are said to serve as sensors and/or actuators that can be used in minimal access or nanoscale surgical procedures with unparalleled precision. [106-108] The computer-guided algorithms of this kind of robotics help the surgeon to precisely guide the robot to a location at a sub-nanometer or nanometer resolution, making it possible to see neurological disease locations that could be impossible to see before. ${ }^{[109]}$ Recently, , a novel image-guided system has been developed for precise, automatic targeting of structures inside the brain. ${ }^{[110,111]}$ The authors reported the robots to either be annexed directly to a head clamp or to the patient skull along the course of surgery (Figure 7). Once placed, it automatically positions itself with great precision. Using programmed information obtained from preoperative electronic scans of the patient, the miniature robots are capable of targeting brain tumors with a precision that has never been before. Similarly, SpineAssist ${ }^{\circledR}$ a miniature bonemounted robotic system for minimally invasive spinal surgery was developed in the same laboratory. ${ }^{[109]}$ The robot is said to have an overall precision of about $1 \mathrm{~mm}$ during pedicle screw in both open and minimally invasive procedures. Another novel nanotechnology that will surely revolutionize the era of minimal access neurosurgical procedures is the "telerobotic surgery" using Robot-Assisted MicroSurgery (RAMS). ${ }^{[112]}$ The integrity and precision of this technology was compared by Le Roux et. al. ${ }^{[113]}$ against the conventional microsurgical techniques in creating and closing carotid arteriotomies in rats. The authors observed the methods to be similar to conventional microsurgical techniques in terms of performance error, precision, and technical quality. [112]

A telecontrolled micromanipulator robotics described as NeuRobot (Figure 8) was used in minimally invasive neurosurgery by Japanese scientists. ${ }^{[114,115]}$

In fact, Goto et. $\mathrm{Al}^{[116]}$ found this robot to be efficient in performing tele-neurosurgery using a computer gated setup as shown in Figure 9.

Previously, the use of nanorobots in intracranial prognosis for brain aneurysm was reported. ${ }^{[117]}$ The main design of these nanorobots is based on a set of integrated circuits block that are capable of identification and tracking the overexpression of protein. And the fabrication techniques were based on three main approaches viz: positional nanoassembly, bacteria nanorobotics and DNA robots. ${ }^{[117]}$ Earlier, a rapidly vibrating micropipette with a tip of less than $1 \mu \mathrm{m}$ in diameter and operation frequency of $100 \mathrm{MHz}$ was used to completely cut apical dendrites from single neurons of rat hippocampal CA1 pyramidal cells without damaging the cell viability. ${ }^{[16,118]}$ Study made by Freitas Jr ${ }^{[19]}$ has envisioned biocompatible surgical nanorobots capable of finding and eliminating isolated cancerous cells. These nanorobots were also said to remove microvascular obstructions and recondition vascular endothelial cells. They can even conduct molecular repairs on traumatized extracellular and intracellular structures, and exchange new whole chromosomes for old ones inside individual living human cells. ${ }^{[119]}$

\section{Conclusion}

It is obvious that the current explosive momentum in nanotechnology research and developments provides a future 


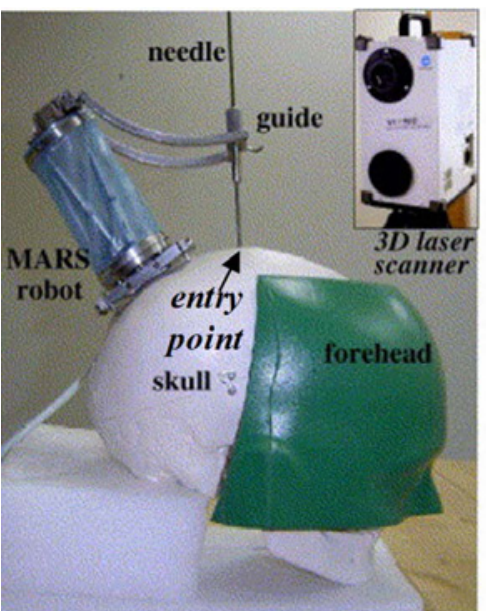

(a) MARS robot mounted on the skull

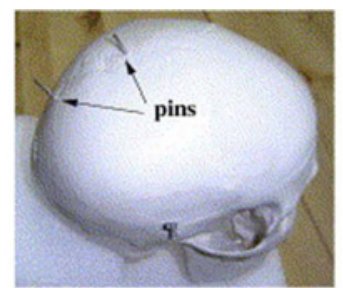

(b) skull-mounted pins

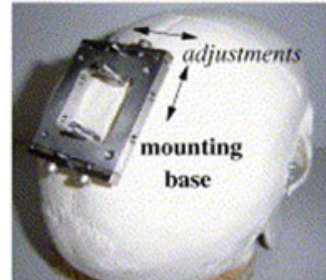

(c) robot mounting base

Figure 7: The Minimal Access Robot-assisted Surgery (MARS) robot mounted on the skull. Adopted from ${ }^{[108]}$ with permission from Elsevier.

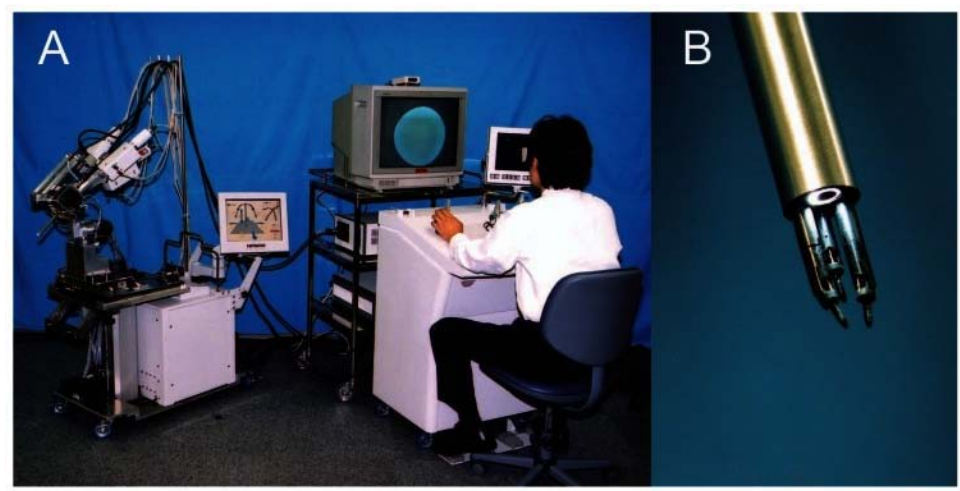

Figure 8: (a) set NeuRobot micromanipulator and the manipulator computer work station, (b) micromanipulator tip with the manipulator arm. Adopted from ${ }^{[116]}$.

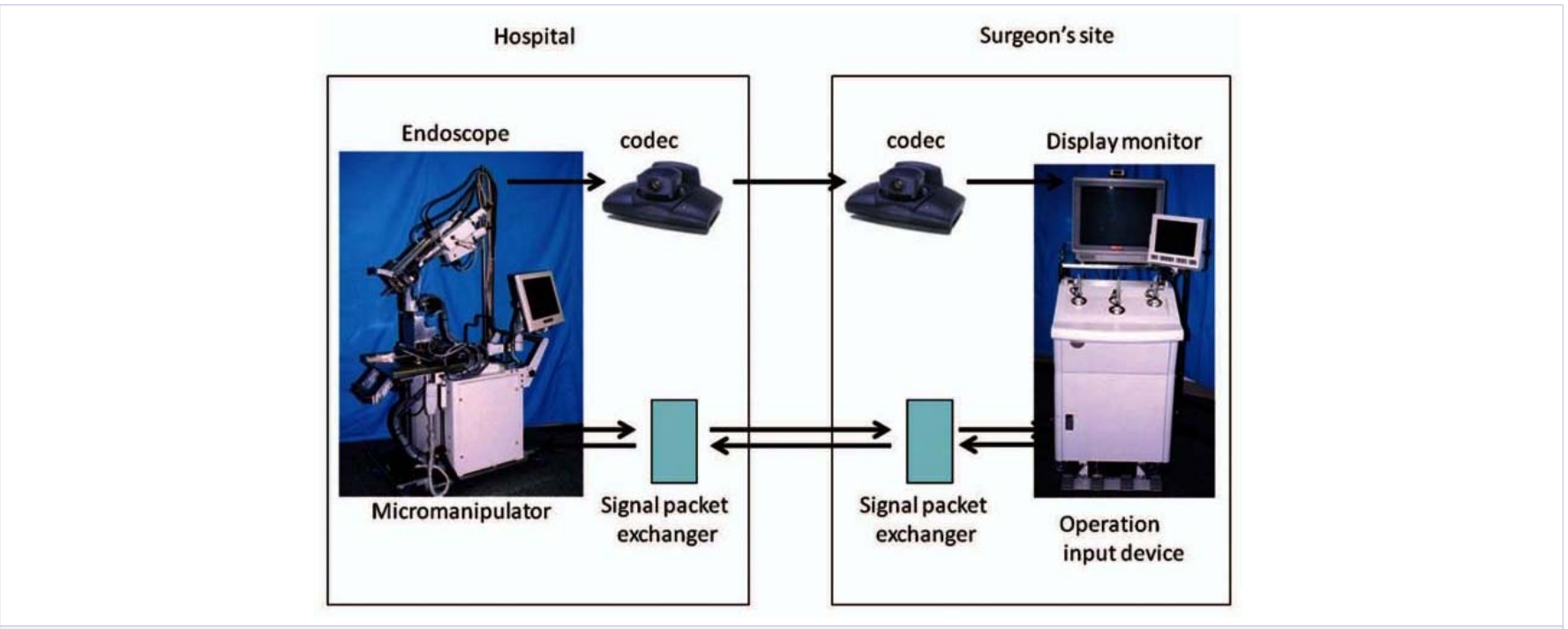

Figure 9: NeuRobot computer gated setup for remote-surgery. The codecs converted the 3D endoscopic images and transmitted the result to a surgeon's site. The operating input device in surgeon's site culled the micromanipulator in a hospital. The packet signal exchanger is responsible for changing, decoding and transmitting the signal between the operation input device and micromanipulator in the hospital. Adopted from ${ }^{[16]}$. 
avenue that portrayed the destiny of neuromedicine and neurosurgery, which will be anticipated to lead to the realization of potential benefits in addressing various difficult conditions of the nervous system encompassing gliomas and other malignant tumors, brain implantation, nerve injuries, efficient drug delivery, novel diagnostic imaging, real-time recording etc. Despite the significant advances provided by nanotechnology in terms of neurosurgical procedures, modern neuroimaging and contrast media; still some significant limitations exist.

With the advent and popularity of minimal access surgeries and micro-medicine techniques, nanotechnology is providing a future platform for further research and development in neuroscience and neurosurgery. Though, nanorobotics have shown some promising potential for solving the imminent problems of invasive neurosurgical techniques, still further research is highly demanded to perfect the procedure for it to be useful in human surgery. Thus, the development of new technologies is therefore needed to advance the capabilities of this important aspect in neurosciences.

\section{References}

1. Muzumdar D. Neurosurgery in the Past and Future. An Appraisal Annals of Medicine and Surgery2012;1:13-15.

2. Sheikh BY, Mohamadin AM. Thymoquinone a potential therapy for cerebral oxidative stress. Asian Journal of Natural and Applied Sciences2012;1(2):76-92.

3. Mohamadin AM, Sheikh B, Abd El-Aal AA, Elberry AA, Al-Abbasi FA Protective effects of Nigella sativa oil on propoxur-induced toxicity and oxidative stress in rat brain regions. Pesticide Biochemistry and Physiology2010; 98(1):128-134.

4. Sheikh BY. Simple practical new instrumentation for holding the neuro-endoscope. Pan Arab Journal of Neurosurgery2014;68-69.

5. Sheikh BY. Minimal invasive method for intraoperative angiography using the superficial temporal artery with preservation of its trunk. Surgical Neurology2008;70(6):640-643.

6. Sheikh B. Safety and efficacy of electrocautery scalpel utilization for skin opening in neurosurgery. Br J Neuro surg2004;18(3):268-272.

7. Sheikh BY, Al Jihani H. Implementing transcranial Doppler as confirmatory test in brain death criteria. Pan Arab Journal of Neurosurgery2008;12(1):46-49.

8. Svenson S, Tomalia DA. Dendrimers in biomedical applications-reflections on the field. Advanced drug delivery reviews $2012 ; 64: 102-115$

9. Fabbro A, Prato M, Ballerini L. Carbon nanotubes in neuroregeneration and repair. Advanced Drug Delivery Reviews2013;65(15):2034-2044.

10. Osawa E. Perspectives of fullerene nanotechnology. Springer2002;400.

11. Han SK, Kim RS, Lee JH, Tae G, Cho SH, Yu HS. Core-Shell Nanoparticles for Drug Delivery and Molecular Imaging. Nanotechnologies for the Life Sciences 2007.

12. Cheng Y, Morshed RA, Auffinger B, Tobias AL, Lesniak MS Multifunctional nanoparticles for brain tumor imaging and therapy. Advanced Drug Delivery Reviews2014; 66(0):42-57.

13. Tavangarian F, Li y. Carbon nanostructures as nerve scaffolds for repairing large gaps in severed nerves. Ceramics International2012;38(8):6075-6090.

14. Del Burgo LS, Hernández RM, Orive G, Pedraz JL. Nanotherapeutic approaches for brain cancer management. Nanomedicine: Nanotechnology, Biology and Medicine2013.

15. Diaz RJ, McVeigh PZ, O’Reilly MA et al. Focused ultrasound delivery of Raman nanoparticles across the blood-brain barrier: Potential for targeting experimental brain tumors. Nanomedicine: Nanotechnology, Biology and Medicine2013.

16. Khawaja AM. The legacy of nanotechnology: Revolution and prospects in neurosurgery. International Journal of Surgery2011;9(8):608-614.

17. Aguilar Z. In: Aguilar Z. ed. Nanomaterials for Medical Applications. Boston: Newnes. 2012.

18. Winer JL, Kim PE, Law M, Liu CY, Apuzzo ML. Visualizing the Future:Enhancing Neuroimaging with Nanotechnology. World Neurosurgery2011;75(5-6):626-637.

19. Pabari A, Yang SY, Seifalian AM, Mosahebi A. Modern surgical management of peripheral nerve gap. Journal of Plastic, Reconstructive \& Aesthetic Surgery2010;63(12):1941-1948.

20. Medintz IL, Medintz, Tetsuo Uyeda H, Ellen R, Goldman, Hedi Mattoussi. Quantum dot bioconjugates for imaging, labelling and sensing. Nature materials2005;4(6):435-446.

21. Ding K, Jing L, Liu C, Hou Y, Gao M. Magnetically engineered Cd-free quantum dots as dual-modality probes for fluorescence/magnetic resonance imaging of tumors. Biomaterials2014;35(5):1608-1617.

22. Bhat AS. NANOBOTS:THE FUTURE OF MEDICINE. International Journal of Engineering and Management Sciences2014;5(1):44-49.

23. Vuilleumier P, Sander D, Baertschi B. Changing the Brain, Changing the Society:Clinical and Ethical Implications of Neuromodulation Techniques in Neurology and Psychiatry. Brain topography2014;27(1):1-3.

24. Fraczek-Szczypta A. Carbon nanomaterials for nerve tissue stimulation and regeneration. Materials Science and Engineering:C2014;34:35-49.

25.Zhang L, Gu FX, Chan JM, Wang AZ, Langer RS, Farokhzad OC. Nanoparticles in medicine:therapeutic applications and developments. Clinical Pharmacology \& Therapeutics2008;83(5):761-769.

26. BBCResearch. Nanotechnology:A Realistic Market Assessment. 2012[online].Available at: http://www.bccresearch.com/marketresearch/nanotechnology/nanotechnology-market-applicationsproducts-nan031e.html.http://www.bccresearch.com/marketresearch/nanotechnology/nanotechnology-market-applicationsproducts-nan031e.html.

27.Wong $\mathrm{HL}$, Wu XY, Bendayan R. Nanotechnological advances for the delivery of CNS therapeutics. Advanced Drug Delivery Reviews2012;64(7):686-700.

28. Wong HL, Bendayan R, Rauth AM, Li Y, Wu XY. Chemotherapy with anticancer drugs encapsulated in solid lipid nanoparticles. Advanced drug delivery reviews2007;59(6):491-504.

29. Malam Y, Loizidou M, Seifalian AM. Liposomes and nanoparticles:nanosized vehicles for drug delivery in cancer. Trends in pharmacological sciences2009;30(11):592-599.

30. Behl C. The search for novel avenues for the therapy and prevention of Alzheimer's disease. Drug News Perspect2006;19(1):5-12.

31. Huber A, Stuchbury G, Bürkle A, Burnell J, Münch G. Neuroprotective Therapies for Alzheimers Disease. Current pharmaceutical 


\section{design2006;12(6):705-717.}

32. Dhawan S, Kapil R, Singh B. Formulation development and systematic optimization of solid lipid nanoparticles of quercetin for improved brain delivery. Journal of Pharmacy and Pharmacology2011;63(3):342-351.

33. Halatsch ME, Schmidt U, Behnke-Mursch J, Unterberg A, Wirtz CR. Epidermal growth factor receptor inhibition for the treatment of glioblastoma multiforme and other malignant brain tumours. Cancer treatment reviews2006;32(2):74-89.

34. Kuo YC, Liang CT. Inhibition of human brain malignant glioblastoma cells using carmustine-loaded catanionic solid lipid nanoparticles with surface anti-epithelial growth factor receptor. Biomaterials2011;32(12):3340-3350.

35. Venishetty VK, Komuravelli R, Kuncha M, Sistla R, Diwan PV. Increased brain uptake of docetaxel and ketoconazole loaded folate-grafted solid lipid nanoparticles. Nanomedicine:Nanotechnology,Biology and Medicine2013;9(1):111-121.

36. Kuo YC, Ko HF. Targeting delivery of saquinavir to the brain using 83-14 monoclonal antibody-grafted solid lipid nanoparticles. Biomaterials2013;34(20):4818-4830.

37. Kumar M, Kakkar V, Mishra AK, Chuttani K, Kaur IP. Intranasal delivery of streptomycin sulfate (STRS) loaded solid lipid nanoparticles to brain and blood. International journal of pharmaceutics2014;461(1):223-233.

38. Kuo YC , Shih-Huang CY. Solid lipid nanoparticles with surface antibody for targeting the brain and inhibiting lymphatic phagocytosis. Journal of the Taiwan Institute of Chemical Engineers2014.

39. Kakkar V, Kaur IP. Evaluating potential of curcumin loaded solid lipid nanoparticles in aluminium induced behavioural,biochemical and histopathological alterations in mice brain. Food and Chemical Toxicology2011;49(11):2906-2913.

40. Patel S, Chavhan S, Soni $\mathrm{H}$ et al. Brain targeting of risperidoneloaded solid lipid nanoparticles by intranasal route. Journal of drug targeting2011;19(6):468-474.

41. Kuo YC, Hong TY. Delivering etoposide to the brain using catanionic solid lipid nanoparticles with surface 5-HT-moduline. International journal of pharmaceutics2014;465(1):132-142.

42. Allard E, Jarnet D, Vessières At et al. Local delivery of ferrociphenol lipid nanocapsules followed by external radiotherapy as a synergistic treatment against intracranial 9L glioma xenograft. Pharmaceutical research2010;27(1):56-64.

43. Ying $X$, Wen $\mathrm{H}$, Lu WL et al. Dual-targeting daunorubicin liposomes improve the therapeutic efficacy of brain glioma in animals. Journal of Controlled Release2010;141(2):183-192.

44. He H, Li Y, Jia XR et al. PEGylated Poly(amidoamine) dendrimerbased dual-targeting carrier for treating brain tumors. Biomaterials2011;32(2):478-487.

45. Huynh NT, Passirani C, Saulnier P, Benoit JP. Lipid nanocapsules: A new platform for nanomedicine. International Journal of Pharmaceutics2009;379(2):201-209.

46. Khalid MN, Simard P, Hoarau D, Dragomir A, Leroux JC. Long circulating poly (ethylene glycol)-decorated lipid nanocapsules deliver docetaxel to solid tumors. Pharmaceutical research2006;23(4):752-758.

47. Stupp R, Mason WP, van den Bent MJ. Radiotherapy plus concomitant and adjuvant temozolomide for glioblastoma. $N$ Engl J Med2005;352(10):987-996.
48. Allard E, Passirani C, Garcion E et al. Lipid nanocapsules loaded with an organometallic tamoxifen derivative as a novel drug-carrier system for experimental malignant gliomas. Journal of Controlled Release 2008;130(2):146-153.

49. Allard E, Hindre F, Passirani C et al. 188Re-loaded lipid nanocapsules as a promising radiopharmaceutical carrier for internal radiotherapy of malignant gliomas. European journal of nuclear medicine and molecular imaging2008;35(10):1838-1846.

50. Béduneau A, Hindré F, Clavreul A, Leroux JC, Saulnier P, Benoit JP. Brain targeting using novel lipid nanovectors. Journal of Controlled Release2008;126(1):44-49.

51.van Rooy I, Mastrobattista E, Storm G, Hennink WE, Schiffelers RM. Comparison of five different targeting ligands to enhance accumulation of liposomes into the brain. Journal of Controlled Release2011;150(1):30-36.

52. Li Y, He H, Jia X, Lu WL, Lou J, Wei Y. A dual-targeting nanocarrier based on poly(amidoamine) dendrimers conjugated with transferrin and tamoxifen for treating brain gliomas. Biomaterials2012;33(15):3899-3908.

53. Hemmer R, Andrew Hall, Robert Spaulding et al. Analysis of Biotinylated Generation 4 Poly (amidoamine)(PAMAM) Dendrimer Distribution in the Rat Brain and Toxicity in a Cellular Model of the Blood-Brain Barrier. Molecules2013;18(9):11537-11552.

54. Huckman MS. Clinical experience with the intravenous infusion of iodinated contrast material as an adjunct to computed tomography. Surgical neurology1975;4(3):297-318.

55. Bourin M, Jolliet p, Ballereau F. An overview of the clinical pharmacokinetics of x-ray contrast media. Clinical pharmacokinetics1997;32(3):180-193.

56. Hainfeld JF, Smilowitz HM, O'Connor MJ, Dilmanian FA, Slatkin DN. Gold nanoparticle imaging and radiotherapy of brain tumors in mice. Nanomedicine2013;8(10):1601-1609.

57.ToddDJ,KayJ.Nephrogenicsystemic fibrosis:anepidemicofgadolinium toxicity. Current rheumatology reports2008;10(3):195-204.

58. Abujudeh HH, Kaewlai R, Kagan A et al. Nephrogenic systemic fibrosis after gadopentetate dimeglumine exposure:case series of 36 patients. Radiology2009;253(1):81-89.

59. Muldoon LL, Sàndor M, Pinkston KE, Neuwelt EA. Imaging, Distribution, and Toxicity of Superparamagnetic Iron Oxide Magnetic Resonance Nanoparticles in the Rat Brain and Intracerebral Tumor. Neurosurgery2005;57(4):785-796.

60. Chertok B, Moffat BA, David AE et al. Iron oxide nanoparticles as a drug delivery vehicle for MRI monitored magnetic targeting of brain tumors. Biomaterials2008;29(4):487-496.

61. Reddy GR, Bhojani MS, McConville P et al. Vascular Targeted Nanoparticles for Imaging and Treatment of Brain Tumors. Clinical Cancer Research2006;12(22):6677-6686.

62. Calvo P, Gouritin B, Villarroya H et al. Quantification and localization of PEGylated polycyanoacrylate nanoparticles in brain and spinal cord during experimental allergic encephalomyelitis in the rat. European journal of neuroscience 2002;15(8):1317-1326.

63. Provenzale J, Silva GA. Uses of nanoparticles for central nervous system imaging and therapy. American journal of neuroradiology2009;30(7):1293-1301.

64. Neuwelt EA, Várallyay P, Bagó AG, Muldoon LL, Nesbit G, Nixon R. Imaging of iron oxide nanoparticles by MR and light microscopy in 
patients with malignant brain tumours. Neuropathology and Applied Neurobiology2004;30(5):456-471.

65. Murillo TP, Chloe Sandquist, Paula M Jacobs, Gary Nesbit, Sandor Manninger, Edward A Neuwelt. Imaging brain tumors with ferumoxtran-10, a nanoparticle magnetic resonance contrast agent. Therapy $2005 ; 2(6): 871-882$.

66. Kopelman R , Yong-Eun Lee Koo, Martin Philbert et al. Multifunctional nanoparticle platforms for in vivo MRI enhancement and photodynamic therapy of a rat brain cancer. Journal of Magnetism and Magnetic Materials2005;293(1):404-410.

67. Cheng Y, Morshed RA, Auffinger B, Tobias AL, Lesniak MS Multifunctional nanoparticles for brain tumor imaging and therapy. Adv drug deliv rev2014;66:42-57.

68. Kim J, Piao Y, Hyeon T. Multifunctional nanostructured materials for multimodal imaging, and simultaneous imaging and therapy. Chemical Society Reviews2009;38(2):372-390.

69. McCarthy JR, Weissleder R. Multifunctional magnetic nanoparticles for targeted imaging and therapy. Advanced drug delivery reviews2008;60(11):1241-1251.

70.Park K, Seulki Lee, Eunah Kang, Kwangmeyung Kim, Kuiwon Choi, Ick Chan Kwon.New generation of multifunctional nanoparticles for cancer imaging and therapy. Advanced functional materials2009;19(10):1553-1566.

71. Kim J, Sungjin Park, Ji Eun Lee et al. Designed fabrication of multifunctional magnetic gold nanoshells and their application to magnetic resonance imaging and photothermal therapy. Angewandte Chemie2006;118(46):7918-7922.

72. Kircher MF, Adam de la Zerda, Jesse V Jokerst et al. A brain tumor molecular imaging strategy using a new triplemodality MRI-photoacoustic-Raman nanoparticle. Nature Medicine2012;18(5):829-834.

73. Kircher MF, Mahmood U, King RS, Weissleder R, Josephson L. A Multimodal Nanoparticle for Preoperative Magnetic Resonance Imaging and Intraoperative Optical Brain Tumor Delineation. Cancer Research2003;63(23):8122-8125.

74. Rhyner MN, Smith AM, Gao X, Mao H, Yang L, Nie S. Quantum dots and multifunctional nanoparticles:new contrast agents for tumor imaging. Nanomedicine2006;1(2):209-217.

75. Santra S, Yang H, Stanley JT et al. Rapid and effective labeling of brain tissue using TAT-conjugated CdS: Mn/ZnS quantum dots. Chemical communications2005;(25):3144-3146.

76. Saito R, Bringas JR, McKnight TR et al. Distribution of liposomes into brain and rat brain tumor models by convection-enhanced delivery monitored with magnetic resonance imaging. Cancer research2004;64(7):2572-2579.

77. Saito R, Krauze MT, Bringas JR et al. Gadolinium-loaded liposomes allow for real-time magnetic resonance imaging of convection-enhanced delivery in the primate brain. Experimental neurology2005;196(2):381-389.

78. Sarin H, Kanevsky AS, Wu H. Effective transvascular delivery of nanoparticles across the blood-brain tumor barrier into malignant glioma cells. Journal of Translational Medicine2008;6(1):80.

79. Yan H, Jiyao Wang, Peiwei Yi et al. Imaging brain tumor by dendrimerbased optical/paramagnetic nanoprobe across the blood-brain barrier. Chemical Communications2011;47(28):8130-8132.

80.Ji X, Ruping Shao, Andrew M Elliott. Bifunctional gold nanoshells with a superparamagnetic iron oxide-silica core suitable for both MR imaging and photothermal therapy. The Journal of Physical Chemistry C2007;111(17):6245-6251.

81. Wang Y, Xueyi Xie,Xueding Wang. Photoacoustic tomography of a nanoshell contrast agent in the in vivo rat brain. Nano Letters2004;4(9):1689-1692.

82. Robinson JT ,Kevin Welsher, Scott M et al. High performance in vivo near-IR $(>1 \mu \mathrm{m})$ imaging and photothermal cancer therapy with carbon nanotubes. Nano Res2010;3(11):779-793.

83. Ren J, Shen S, Wang D et al. The targeted delivery of anticancer drugs to brain glioma by PEGylated oxidized multi-walled carbon nanotubes modified with angiopep-2. Biomaterials2012;33(11):3324-3333.

84. Gianella A, Jarzyna PA, Mani V. Multifunctional nanoemulsion platform for imaging guided therapy evaluated in experimental cancer. ACS nano2011;5(6):4422-4433.

85. Rapoport N, Nam KH, Gupta R et al. Ultrasound-mediated tumor imaging and nanotherapy using drug loaded, block copolymer stabilized perfluorocarbon nanoemulsions. Journal of Controlled Release2011;153(1):4-15.

86. Manchester M, Singh P. Virus-based nanoparticles (VNPs):platform technologies for diagnostic imaging. Advanced drug delivery reviews2006;58(14):1505-1522.

87. Matsumoto K, Shimizu N. Activation of the phospholipase C signaling pathway in nerve growth factor-treated neurons by carbon nanotubes. Biomaterials;34(24):5988-5994.

88. Aguilar ZP. Nanomedical Devices. In: Aguilar ZP ed. Nanomaterials for Medical Applications. New York: Elsevier, 2013. p. 235-292.

89. Tabatabaei SN, editor. Towards MR-navigable nanorobotic carriers for drug delivery into the brain. In: Robotics and Automation (ICRA). IEEE International Conference; 2012 14-18 May; Saint Paul, USA. IEEE; 2012.

90. Khalil ISM, Magdanz V, Sanchez S, Schmidt OG, Abelmann L, Misra S. Magnetic control of potential microrobotic drug delivery systems: Nanoparticles, magnetotactic bacteria and self-propelled microjets. In Engineering in Medicine and Biology Society (EMBC). 35th Annual International Conference of the IEEE; 2013 3-7 July 2013; Osaka. IEEE; 2013.

91. Martel S. Therapeutic Bacterial Nanorobots for Targeted Drug Delivery Deep Inside Tumors. In: Mavroidis C, Ferreira A eds. Nanorobotics. New York: Springer, 2013. p. 323-329.

92. Suter M, Zhang L, Siringil EC et al. Superparamagnetic microrobots: fabrication by two-photon polymerization and biocompatibility. Biomedical microdevices2013;15(6):997-1003.

93. Hassan S, Ikram Ullah, Myeong Ok Kim, Jungwon Yoon. Neuro Invasive Multi-targeted Drug Delivery Approach Using Swarm of Nano-robotic Carriers. In: Min Cheol Lee, Honghai Liu, Jee-Hwan Ryu, eds. Intelligent Robotics and Applications. Berlin Heidelberg: Springer, 2013. p. 204215.

94. LaVan DA, McGuire T, Langer R. Small-scale systems for in vivo drug delivery. Nat Biotech2003;21(10):1184-1191.

95. Couvreur P, Vauthier C. Nanotechnology:Intelligent Design to Treat Complex Disease. Pharmaceutical Research2006;23(7):1417-1450.

96. Ben-Yakar A, Bourgeois F. Ultrafast laser nanosurgery in microfluidics for genome-wide screenings. Current Opinion in Biotechnology2009;20(1):100-105. 
97. Nguyen J, Ferdman J, Zhao M et al. Sub-surface, micrometer-scale incisions produced in rodent cortex using tightly-focused femtosecond laser pulses. Lasers in Surgery and Medicine2011;43(5):382-391.

98. Eversole DS. Plasmonic laser nanosurgery2013.

99. Hoy CL, Ferhanoglu O, Yildirim M et al. Clinical ultrafast laser surgery: recent advances and future directions. Selected Topics in Quantum Electronics, IEEE Journal of 2014;20(2):1-14.

100. Vogel A, Noack J, Hüttman G, Paltauf G. Mechanisms of femtosecond laser nanosurgery of cells and tissues. Applied Physics B2005;81(8):1015-1047.

101. Jain K. Nanoneurology. In: Applications of Biotechnology in Neurology. New York: Humana Press, 2013. p. 563-574.

102. Sheikh B, Raghed Atta, Mostafa Abdal Moneim. Chronic Brain cortical recording using siliconsubstrate microelectrode arrrays implanted in cerebral cortex International. Journal of Academic Research2011;3(3).

103. Qiao Y, Chen J, Guo X, Cantrell D, Ruoff R, Troy J. Fabrication of nanoelectrodes for neurophysiology:cathodic electrophoretic paint insulation and focused ion beam milling. Nanotechnology2005;16(9):1598-1602.

104. Kim W, Ng JK, Kunitake ME, Conklin BR, Yang P. Interfacing silicon nanowires with mammalian cells. Journal of the American Chemical Society2007;29(23):7228-7229.

105. Robinson JT, Marsela Jorgolli, Alex k. Shalek. Vertical nanowire electrode arrays as a scalable platform for intracellular interfacing to neuronal circuits. Nature nanotechnology2012;7(3):180-184.

106. Ahmadi SA editor. Advanced planning and intra-operative validation for robot-assisted keyhole neurosurgery In ROBOCAST. In: Advanced Robotics. International Conference IEEE; 22-26 June 2009; Munich, Germany. IEEE; 2009

107. De Momi E, Ferrigno G. Robotic and artificial intelligence for keyhole neurosurgery: the ROBOCAST project, a multi-modal autonomous path planner. Proceedings of the Institution of Mechanical Engineers, Part H: Journal of Engineering in Medicine2010;224(5):715-727.

108. Joskowicz L, Shohamb M, Shamira R, Freimana M, Zehavic E, Shoshan
Y. Miniature robot-based precise targeting system for keyhole neurosurgery: concept and preliminary results. In International Congress Series; New York, Elsevier. 2005

109. Shoham M, Lieberman IH, Benzel EC. Robotic assisted spinal surgery-from concept to clinical practice. Computer Aided Surgery2007;12(2):105-115.

110. Joskowicz L, Shamir R, Freiman M et al. Image-guided system with miniature robot for precise positioning and targeting in keyhole neurosurgery. Computer Aided Surgery2006;11(4):181-193.

111. Shamir, R, M Freiman, L Joskowicz, M Shoham, E Zehavi, Y Shoshan. Robot-assisted image-guided targeting for minimally invasive neurosurgery: intraoperative robot positioning and targeting experiment. In MICCAI Workshop Proceedings; Citeseer. 2006

112. Liverneaux PA, Berner SH, Bednar MS et al. Telemicrosurgery: robot assisted microsurgery. New York: Springer. 2012.

113. LeRouxPD,Das H,EsquenaziS, KellyPJ.Robot-assisted Microsurgery:A Feasibility Study in the Rat. Neurosurgery2001;48(3):584-589.

114. Hongo K, T Goto, T Miyahara, Y Kakizawa, J Koyama, Y Tanaka et al. Telecontrolled micromanipulator system (NeuRobot) for minimally invasive neurosurgery. In Medical Technologies in Neurosurgery. New York: Springer. 2006. p. 63-66.

115. Hongo K, Kobayashi S, Kakizawa Y et al. NeuRobot:telecontrolled micromanipulatorsystem forminimallyinvasivemicroneurosurgerypreliminary results. Neurosurgery2002;51(4):985-988.

116. Goto T, Takahiro Miyahara, Kazutaka Toyoda. Telesurgery of microscopic micromanipulator system "NeuRobot" in neurosurgery:interhospital preliminary study. Journal of Brain Disease2009;1:45-53.

117. Cavalcanti A. Nanorobot for Brain Aneurysm. The International Journal of Robotics Research2009;28(4):558-570.

118. Kirson ED, Yaari Y. A novel technique for micro-dissection of neuronal processes. Journal of neuroscience methods2000;98(2):119-122.

119. Freitas Jr RA. Nanomedicine, Vol. IIA: Biocompatibility. Georgetown:Landes Bioscience2003;330. 